\title{
Undulator commissioning by characterization of radiation in x-ray free electron lasers
}

\author{
Takashi Tanaka, ${ }^{*}$ Shunji Goto, Toru Hara, Takaki Hatsui, Haruhiko Ohashi, Kazuaki Togawa, \\ Makina Yabashi, and Hitoshi Tanaka \\ RIKEN SPring-8 Center, Koto 1-1-1, Sayo, Hyogo 679-5148, Japan
}

(Received 21 September 2012; published 12 November 2012)

\begin{abstract}
In x-ray free electron lasers (XFELs) where a long undulator composed of many segments is installed, there exist a number of error sources to reduce the FEL gain such as the trajectory error, $K$ value discrepancy, and phase mismatch, which are related to the segmented-undulator structure. Undulator commissioning, which refers to the tuning and alignment processes to eliminate the possible error sources, is thus an important step toward realization of lasing. In the SPring-8 angstrom compact free electron laser (SACLA) facility, the undulator commissioning has been carried out by means of characterization of $\mathrm{x}$-ray radiation, i.e., measurements of the spatial and spectral profiles of monochromatized spontaneous undulator radiation as well as by probing the FEL intensity. The achieved tuning and alignment accuracies estimated from the statistics of actual measurements in SACLA show the effectiveness of this commissioning scheme.
\end{abstract}

DOI: 10.1103/PhysRevSTAB.15.110701

PACS numbers: 07.85.Qe, 41.60.Ap, 41.60.Cr

\section{INTRODUCTION}

In x-ray free electron lasers (XFELs) such as the Linac Coherent Light Source (LCLS) [1] and the SPring-8 angstrom compact free electron laser (SACLA) [2], a high quality electron beam with a small transverse emittance and a high peak current is required and thus the commissioning of many accelerator components such as the electron gun, rf cavities, and dipole and quadrupole magnets has to be performed to finely tune the beam parameters. It should be noted, however, that this is not the final step toward realization of lasing. We have to make sure that the undulator, in which the FEL amplification process takes place, works properly.

In general, the length of the undulator installed in XFEL facilities is of the order of $100 \mathrm{~m}$ to reach saturation in an angstrom-wavelength region. From the practical point of view, such a long undulator is usually divided into many segments not only for fabrication feasibility but also to install monitors for electron diagnostics and magnets for trajectory correction and betatron matching. Because of such a segmented structure, there exist many error sources that can lead to FEL gain reduction even if each undulator segment is perfect. In addition, the wakefield effect can be serious especially in SACLA where narrow-gap in-vacuum undulator (IVUs) are in operation. In order to correct or compensate all these errors and effects, we have to align components or tune devices related to undulator operation and optimize a number of parameters so that

\footnotetext{
*ztanaka@spring8.or.jp
}

Published by the American Physical Society under the terms of the Creative Commons Attribution 3.0 License. Further distribution of this work must maintain attribution to the author(s) and the published article's title, journal citation, and DOI. all the undulator segments work coherently and behave as a single device. Such a process of alignment or tuning is simply referred to as "alignment" or "align," hereinafter in this paper, and the optimization procedure based the alignment processes is referred to as undulator commissioning in contrast to the accelerator commissioning. In SACLA, the undulator commissioning has been carried out by means of characterization of spontaneous radiation as well as probing the FEL intensity.

In the following sections, the error sources that affect the FEL amplification process are explained and their tolerances are given, which are estimated by numerical studies. Then details of the commissioning procedures to correct these errors are presented together with the actual results obtained in SACLA. Finally, achieved alignment accuracies are evaluated to show the effectiveness of this commissioning scheme.

\section{ERROR SOURCES TO REDUCE THE FEL GAIN}

Before describing the details of undulator commissioning based on characterization of radiation, let us first mention the error sources that affect the FEL gain. We have mainly three sources related to the long undulator composed of more than one segment: trajectory error, $K$-value discrepancy, and phase mismatch. In addition, wakefield-induced energy loss is another point to be concerned in SACLA, where narrow-gap IVUs are used.

\section{A. Trajectory error}

The trajectory straightness is one of the most important points for realization of XFELs. If the electron moves along a trajectory deviated from a straight line, the FEL gain is significantly reduced because of the mechanisms explained in $[3,4]$. 
The trajectory error can be classified into two types. One is the random trajectory error or trajectory wander within a single undulator segment, which comes from the localized field error intrinsic to imperfections of permanent magnets. The other, which is more global, is the inclined beam injection into each undulator segment. The former can be corrected by a precise field measurement followed by a conventional undulator field correction technique. In fact, it is possible to reduce the trajectory error within a negligible level using the state-of-the-art technology. The latter is in principle corrected by steering the electron beam so that it goes through the origins of beam position monitors (BPMs) that are aligned in line. It should be noted, however, that the alignment accuracy of BPMs with a conventional survey method is usually insufficient to achieve lasing, especially in the angstrom-wavelength region because the tolerance of the trajectory straightness becomes more stringent for shorter wavelengths [4].

\section{B. $K$-value discrepancy}

Because the lasing wavelength depends on the undulator $K$ value, its discrepancy between segments results in the FEL gain reduction. Roughly speaking, the FEL gain bandwidth is nearly equal to the so-called Pierce parameter $\rho$, within which the wavelength deviation should be.

It is well known that the mathematical form of the vertical magnetic field $B_{y}$ in a planar permanent magnet undulator with the Halbach configuration under the twodimensional approximation is given as [5]

$$
B_{y}(g, y, z)=B_{0} \exp \left(-k_{u} g / 2\right) \cosh \left(k_{u} y\right) \sin \left(k_{u} z\right)
$$

with

$$
k_{u}=2 \pi / \lambda_{u},
$$

where $y$ and $z$ are the vertical and longitudinal coordinates, $B_{0}$ is the maximum peak magnetic field at the gap of 0 , and $g$ is the undulator gap. The above formula means that the undulator $K$ value is a function of not only the gap but also the vertical offset between the electron beam and the magnetic center $(y=0)$. Thus, we have two error sources resulting in the $K$-value discrepancy between undulator segments, i.e., the gap deviation and height misalignment, both of which should be corrected in order to finely tune the undulator $K$ values.

\section{Phase mismatch}

In order to keep the electrons and photons in phase over the whole undulator length, the phase matching condition should be satisfied, i.e., the phase slippage in the drift section should be a multiple of $2 \pi$. Because the phase slippage is a function of the wavelength of radiation, it needs to be tuned when changing the undulator gap and laser wavelength. The phase shifter is a magnetic device for this purpose, which is functionally equal to a one- period undulator and is installed in every drift section. If the phase shifter is not well tuned and the phase matching condition is violated, the FEL gain is significantly reduced.

\section{Wakefield}

The wakefield is an electromagnetic field generated by interaction between the electron beam and surrounding object. In XFELs, the correlated energy variation along the electron bunch, which is induced by the resistive wakefield, can lead to the FEL gain reduction. Because the strength of the wakefield is reciprocally proportional to the square of the distance from the electron to the surrounding object, this problem can be more serious for narrow-gap IVUs as in SACLA. The undulator tapering, i.e., changing the $K$ value linearly from segment to segment, is an effective way of compensating this effect.

\section{SPECIFICATION OF ALIGNMENT TOLERANCE}

In order to suppress the FEL gain reduction due to the error sources explained in the previous sections, respective components related to undulator operation should be well aligned. In order to look for the alignment tolerances, numerical studies have been performed as explained in the following sections.

\section{A. Estimation of gain reduction}

The gain reductions due to respective error sources have been estimated by means of FEL simulations performed with the code SIMPLEX [6] using the electron beam and undulator parameters in SACLA as summarized in Table I. Note that the electron beam parameters have been estimated through comparisons between the measured and calculated gain curves [2]. As an example, the process of

TABLE I. Electron beam and undulator parameters used in the FEL simulations to specify the alignment tolerance.

\begin{tabular}{lc}
\hline \hline Parameter & Value \\
\hline Electron beam & \\
Energy & $8 \mathrm{GeV}$ \\
Peak current & $3.5 \mathrm{kA}$ \\
Bunch length (FWHM) & $20 \mathrm{fsec}$ \\
Slice emittance & $\pi .7 \mathrm{~mm} \mathrm{mrad}$ \\
Energy spread & $10^{-4}$ \\
Average betatron value & $30 \mathrm{~m}$ \\
Undulator & \\
Period & $18 \mathrm{~mm}$ \\
$K$ value & 2.1 \\
Gap & $3.7 \mathrm{~mm}$ \\
Segment length & $5 \mathrm{~m}$ \\
Drift length & $1.15 \mathrm{~m}$ \\
Number of segments & 18 \\
\hline \hline
\end{tabular}


how to estimate the gain reduction by the trajectory error is explained as follows.

For simplicity, the electron trajectory is assumed to go through the origins of BPMs installed in the drift sections between undulator segments. Namely, the horizontal and vertical beam positions $x_{i}$ and $y_{i}$ at the $i$ th drift section are given by

$$
x_{i}=R_{i} \sigma_{x}
$$

and a similar expression for $y_{i}$, where $R_{i}$ means a Gaussian random number with zero mean and unit variance and $\sigma_{x}$ denotes the standard deviation of BPM positional error, which can be also regarded as the alignment accuracy of the BPM origin. Then, the FEL pulse energy is calculated with the simulation taking the trajectory error into account. This numerical process is repeated by 100 times with changing the error condition, i.e., the seed number of the random number generator. One example of the calculation result in the case when $\sigma_{x, y}=10 \mu \mathrm{m}$ is shown in Fig. 1 as the histogram of the pulse energy. Note that the expected pulse energy without any trajectory error is about $0.19 \mathrm{~mJ}$ as indicated by the dashed line in the same figure. Compared to this ideal value, the pulse energy with $\sigma_{x, y}$ of $10 \mu \mathrm{m}$ is found to decrease significantly and fluctuate with the initial seed number, i.e., the alignment condition of the BPMs.

The gain reduction can be estimated by calculating the average and standard deviation of the pulse energy as a function of $\sigma_{x, y}$ by repeating the above process, the result of which is shown in Fig. 2. Note that $\sigma_{x}$ and $\sigma_{y}$ are assumed to be the same, meaning that the alignment accuracy of the BPM is isotropic.

Besides the BPM positional deviation, it is possible to specify the trajectory error by injection angles $x_{i}^{\prime}$ and $y_{i}^{\prime}$ of

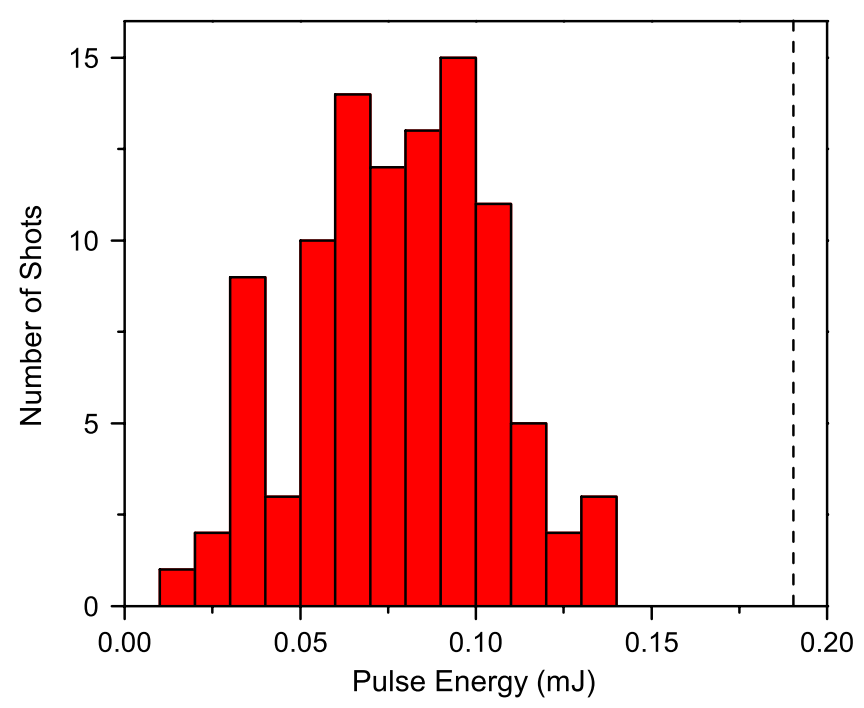

FIG. 1. Histogram of the simulated FEL pulse energy under the condition $\sigma_{x, y}=10 \mu \mathrm{m}$.

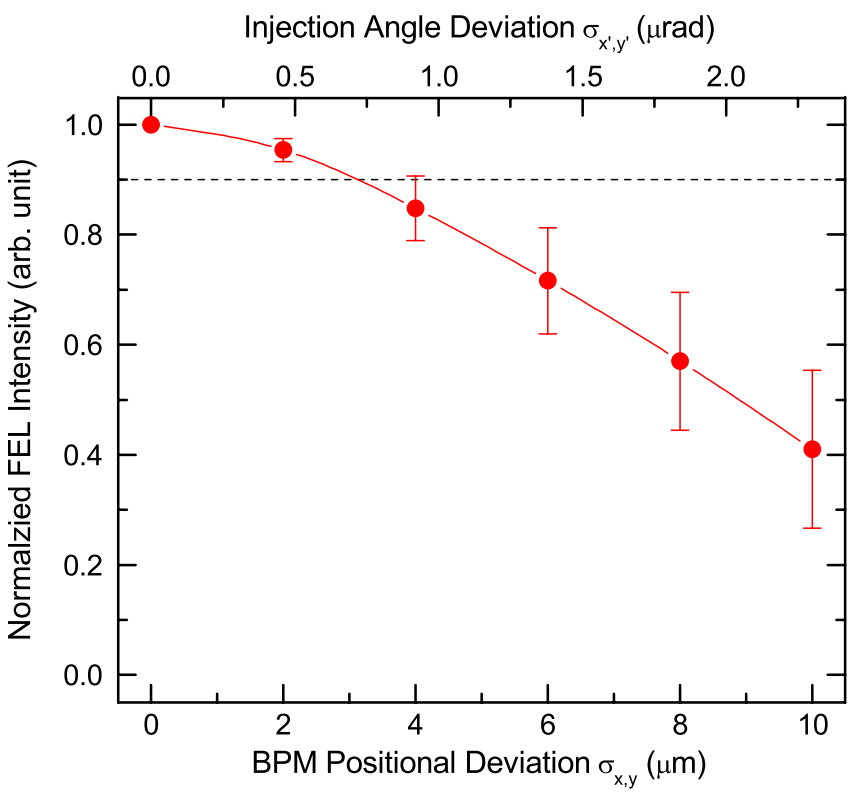

FIG. 2. Estimation of the gain reduction due to trajectory errors. In each error condition, the FEL pulse energy averaged over 100 error models is plotted with the standard deviation as error bars.

the electron beam to the $i$ th undulator segment, which is defined as

$$
x_{i}^{\prime}=\frac{x_{i}-x_{i-1}}{D},
$$

and a similar expression for $y_{i}^{\prime}$, where $D$ is the distance between two adjacent BPMs. The standard deviations of the injection angles, $\sigma_{x^{\prime}, y^{\prime}}$, are then given by

$$
\sigma_{x^{\prime}, y^{\prime}}=\frac{\sqrt{2} \sigma_{x, y}}{D}
$$

For reference, $\sigma_{x^{\prime}, y^{\prime}}$ corresponding to $\sigma_{x, y}$ are indicated in the top scale in Fig. 2. As explained later, the injection angles of the electron beam are measured instead of the positional errors of BPMs for the trajectory alignment in SACLA.

\section{B. Specification of acceptable tolerance}

Based on the estimation of the gain reduction described in the previous section, the acceptable tolerances of respective alignment processes have been specified. As an example, the procedure of how to specify the trajectory alignment tolerance is presented in the following.

As shown in Fig. 2, the average pulse energy $\langle\mathcal{E}\rangle$ over 100 error models decreases with the increased alignment error, while the standard deviation $\Sigma_{\mathcal{E}}$ becomes larger. Thus, it is reasonable to put an upper limit on the alignment tolerance so that the pulse energy $\langle\mathcal{E}\rangle-2 \Sigma_{\mathcal{E}}$ should be higher than a given percentage of the ideal pulse energy. It should be emphasized that, by subtracting the term $2 \Sigma_{\mathcal{E}}$, 
TABLE II. Target alignment items for the undulator commissioning and requirements on the alignment accuracy determined through the numerical study based on FEL simulations. The achieved accuracies in SACLA estimated from the measured statistics are also indicated (refer to Sec. V). The taper is given as the reduction of the $K$ value per segment.

\begin{tabular}{lccc}
\hline \hline Target Item & Required & $\begin{array}{c}\text { Accuracy } \\
\text { Achieved }\end{array}$ & Unit \\
\hline Trajectory & & $\ldots$ & \\
BPM & 2.2 & $0.22(x)$ & $\mu \mathrm{m}$ \\
Injection angle & 0.65 & $0.48(y)$ & \\
$K$ value & & & \\
Total & 5.0 & 1.8 & $10^{-4}$ \\
Gap & 1.9 & 0.6 & $\mu \mathrm{m}$ \\
Height & 60 & 10 & $\mu \mathrm{m}$ \\
Phase slippage & 30 & 15 & degree \\
Taper/segment & $10^{-4}$ & $4 \times 10^{-5}$ & \\
\hline \hline
\end{tabular}

the probability of getting the pulse energy larger than the acceptable level is improved from $50 \%$ to $97.5 \%$, which is easily understood from the characteristics of Gaussian distribution.

Based on the above discussion, the acceptable tolerances for respective error sources have been determined so that the FEL intensity loss due to each error source is at least less than $10 \%$. The results are summarized in Table II, which can be regarded as the required accuracies of the individual alignment procedures in the undulator commissioning. The $K$ value deviation, which comes from the misalignment of both the gap and height of the undulator, should be less than $5 \times 10^{-4}$ in total. Based on the magnetic measurement of the SACLA undulator, this number can be converted to the alignment tolerances of $1.9 \mu \mathrm{m}$ in gap and $60 \mu \mathrm{m}$ in height. Also note that the trajectory alignment accuracy is given both in terms of the BPM position and injection angle of the electron beam.

\section{METHODS AND RESULTS OF COMMISSIONING}

In order to align the components within the required accuracies as summarized in Table II, characterization of spontaneous radiation and probing the FEL intensity have been carried out in SACLA, the details of which are presented in the following sections.

\section{A. Photon diagnostics}

The photon diagnostics system in SACLA is schematically illustrated in Fig. 3. The slit assembly placed $80 \mathrm{~m}$ far from the exit of the last (18th) undulator is used for shaping the photon beam, whose aperture size is variable and selected according to the purpose of measurement. The FEL radiation pulse energy is measured by the photon BPM [7] installed behind the slit, which also works as a photon intensity monitor. Then, the photon beam is monochromatized by a double-crystal monochromator and detected by a photodiode to measure the photon flux, or by a two-dimensional $\mathrm{x}$-ray profiler to measure the spatial profile.

\section{B. Trajectory alignment}

In order to perform the trajectory alignment based on the BPMs installed in the drift sections, all of them should be well aligned in line within a tight tolerance of several microns. The electron beam based alignment method [8], which is routinely carried out in LCLS for trajectory alignment, is a procedure to estimate the BPM offsets with respect to a fiducial line, through the response of the BPM readings to the change of the electron energy. Although this method gives an excellent result of achieved trajectory straightness below $5 \mu \mathrm{m}$ over the 132-m long undulator line [9], it requires a number of steps such as changing the electron energy, taking many BPM readings, and data analysis based on a complex numerical method. In addition, the BPMs should be highly reliable in terms of the resolution, repeatability, and linearity.

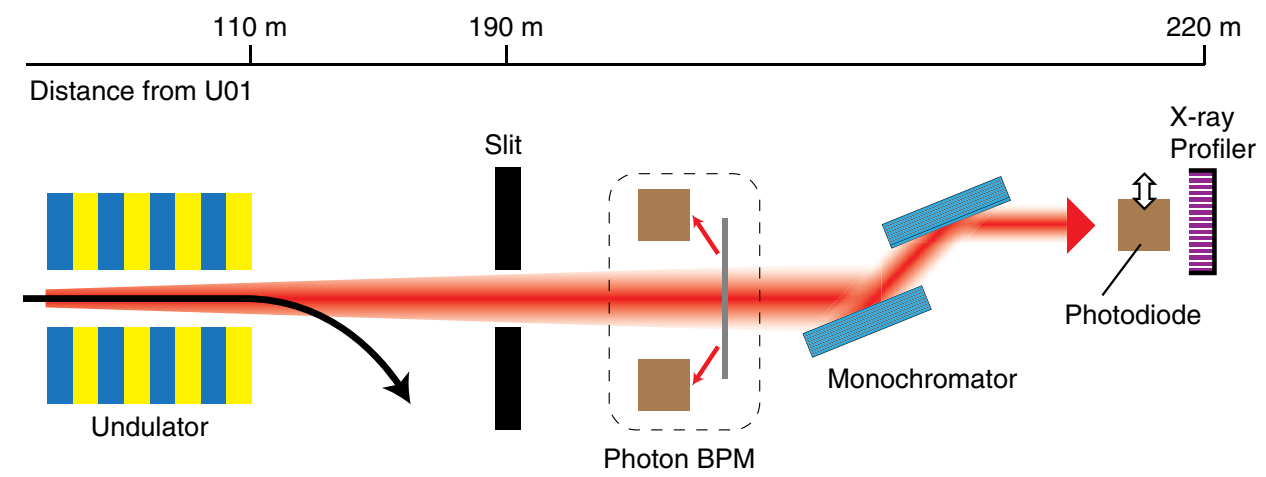

FIG. 3. Schematic illustration of the photon diagnostics system in SACLA. The top scale shows the distance from the entrance of the 1st undulator segment (U01). 
In SACLA, a more simple and straightforward approach has been taken: the angle of beam injection to a specific undulator segment is estimated from the measurement of the spatial profile of spontaneous undulator radiation (UR). The key point in this method is the capability of the $\mathrm{x}$-ray profiler because the radiation is monochromatized with a bandwidth of $10^{-4}$ to reduce the beam size, which results in a significant reduction of the photon counts. In the early stage of undulator commissioning, a multiport CCD detector having photon counting capability and a high spatial resolution was chosen as the $\mathrm{x}$-ray profiler, which has been originally developed for imaging experiments at SACLA [10]. After the confirmation of the detector requirements, it was replaced with a cost-effective microchannel-plate detector with lower detection efficiency. With either of the two detectors, a clear image of monochromatized spontaneous UR from a single undulator segment has been obtained with a single-shot measurement, as indicated in Fig. 4, in which the spontaneous UR was monochromatized at the photon energy of $10 \mathrm{keV}$ and the pulse energy reaching the x-ray profiler was nearly $0.4 \mathrm{~nJ}$. Note that the slit is fully opened in this process to take the image as wide as possible.

The centroid of the measured spatial profile reflects the injection angle of the electron beam. It is therefore possible to correct the trajectory error by adjusting the injection angle so that all the spatial profiles of spontaneous UR from individual undulator segments have the identical centroids. What is important in this procedure is the pointing stability of the monochromatized spontaneous UR.

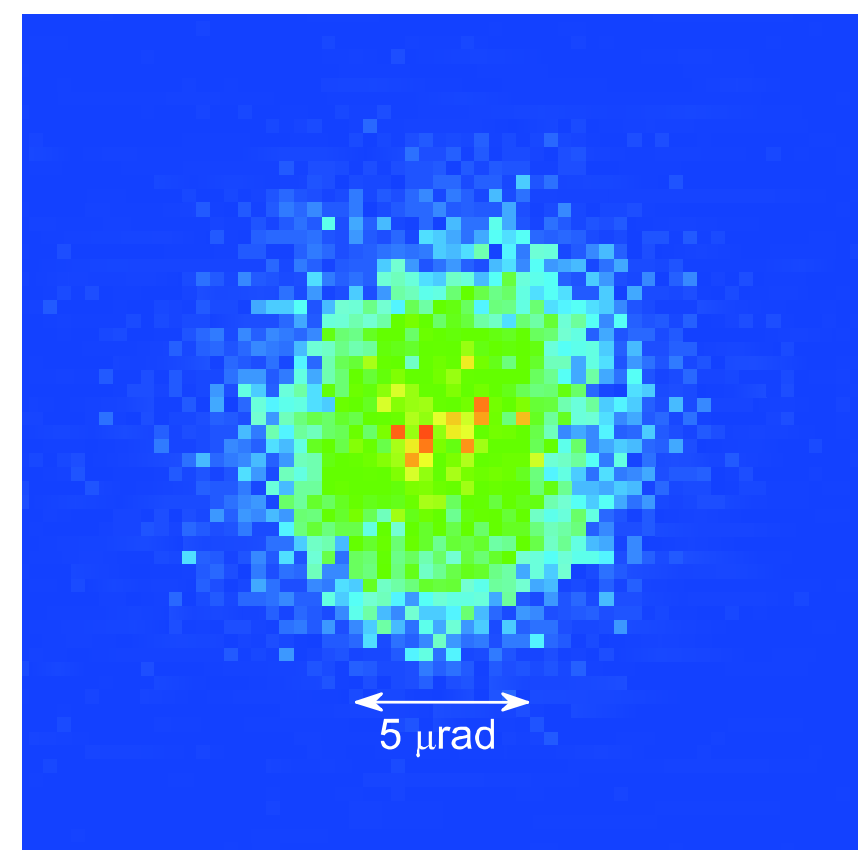

FIG. 4. Example of a single-shot image of monochromatized spontaneous UR from the 1st undulator segment, taken by the $\mathrm{x}-$ ray $\mathrm{CCD}$ detector.
Figure 5 shows the example of the pointing stability measured over 100 shots. The standard deviations have been found to be 0.22 and $0.48 \mu \mathrm{rad}$ in the horizontal and vertical directions, respectively, being less than the alignment tolerance of $0.50 \mu \mathrm{rad}$. It is thus feasible to apply this procedure for the trajectory alignment, at least in SACLA.

Once the reference trajectory is determined at a certain gap with the above procedure, the trajectory deviation from the reference is corrected by two steering magnets installed at the both ends of each undulator segment, which ensures the trajectory straightness at other gap values.

\section{C. $K$-value tuning}

It is well known that the spontaneous UR is quasimonochromatic around the so-called fundamental energy $\omega_{1}$ given by

$$
\omega_{1}=\frac{4 \pi c \gamma^{2}}{\lambda_{u}\left(1+K^{2} / 2+\gamma^{2} \theta^{2}\right)},
$$

where $c$ is the speed of light, $\gamma$ is the Lorentz factor of the electron, $\lambda_{u}$ is the undulator period, $K$ is the undulator $K$ value, and $\theta$ is the radial angle of observation with respect to the undulator axis. It is thus possible to estimate the undulator $K$ value by measuring the spectrum of spontaneous UR and detecting the peak energy. Because of the dependence of the fundamental energy on the observation angle, the spectral bandwidth becomes wider for larger angular acceptance. Thus, it seems reasonable to keep the slit aperture size as small as possible for a better resolution. Contrary to this general approach, the slit is fully opened to increase the angular acceptance during the

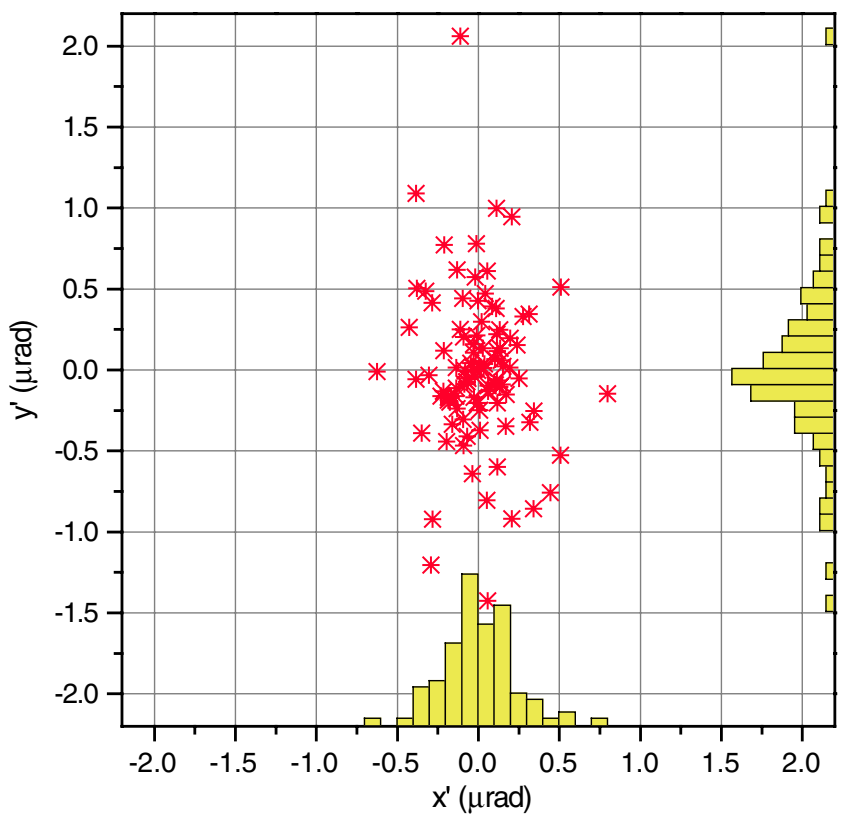

FIG. 5. Example of the pointing stability of the monochromatized spontaneous UR. 
$K$-value tuning in SACLA, the reason for which is explained in the following.

The photon flux of spontaneous UR is a function of many parameters related to the electron beam quality and observation condition, namely, it is expressed as

$$
f\left(\hbar \omega, K, L, \Delta X, \Delta Y, E, \sigma_{e}, x, y, x^{\prime}, y^{\prime}, \sigma_{x}, \sigma_{y}, \sigma_{x^{\prime}}, \sigma_{y^{\prime}}\right),
$$

where $\hbar \omega$ is the photon energy, $L$ is the distance from the undulator to the slit located in front of the monochromator, $\Delta X$ and $\Delta Y$ are the horizontal and vertical apertures of the slit, $E$ and $\sigma_{E}$ are the average energy and energy spread of the injected electron beam, $x, x^{\prime}, \sigma_{x}, \sigma_{x^{\prime}}$ are the horizontal position, angle, beam size, and angular spread of the electron beam, and similar notations in the vertical direction. In this manner, the UR spectrum depends on a huge number of parameters.

Recalling the fact that the electron beam property in the linear accelerator can fluctuate shot by shot, it is important to reduce the number of parameters affecting the alignment process. In addition, the fact that the photon flux is a function of the slit conditions $L, \Delta x$, and $\Delta y$ is also troublesome when repeating the $K$-value tuning process from segment to segment, because $L$ is a function of the segment number. We have to adjust the slit sizes $\Delta x$ and $\Delta y$ according to the target segment number so that the angular acceptance is kept constant.

Now let us consider the case when the slit aperture is opened so that the angular acceptance is wider than the angular spread of spontaneous UR and thus is effectively equal to the whole solid angle. The photon flux then becomes insensitive to the slit condition, $L, \Delta X$, and $\Delta Y$, and to the electron beam quality in the four-dimensional phase space, $x, y, x^{\prime}, y^{\prime}, \sigma_{x}, \sigma_{y}, \sigma_{x^{\prime}}$, and $\sigma_{y^{\prime}}$. The expression for the photon flux is now simplified to

$$
f\left(\hbar \omega, K, E, \sigma_{e}\right),
$$

which contains just four parameters. The $K$ value can be accurately determined through the spectral measurement regardless of the segment number, beam emittance, Twiss parameters, and injection conditions. The resolution and accuracy depends on the energy spread and stability of the beam energy. The drawback of adopting the wide aperture is that the spectral bandwidth is broadened and a simple peak-detection method does not work.

Figure 6(a) shows the variation in spectrum with horizontal injection angle when the slit aperture is opened to $10 \mathrm{~mm}$ in both directions, which has been calculated with the spontaneous synchrotron radiation calculation code SPECTRA [11]. As expected from the above discussions, the variation is found to be negligible. This means that we will be able to get the same measurement results regardless of the beam condition as long as the electron energy and energy spread are kept constant. On the other hand, the calculation results with the aperture size of $0.5 \mathrm{~mm}$ shown in Fig. 6(b) suggests that the spectral shape and peak position depend largely on the injection angle. In conclusion, wider aperture is more reasonable in the process of $K$-value tuning. It should be noted, however, that we have to establish a procedure to specify the $K$ value from the step-function-like spectral profile as shown in Fig. 6(a), but not from a simple Gaussian-like profile as in Fig. 6(b).

As mentioned in Sec. II B, the undulator should be well aligned both in terms of the gap and height to finely tune the $K$ value. In the following sections, the detailed procedures in respective alignment steps are presented.

\section{Gap distance adjustment}

In order to align the undulator gap, photon flux at a specific photon energy is measured as a function of the gap. The higher energy edge of the spectral profile (spectral edge) that corresponds to the undulator fundamental energy $\omega_{1}$ is shifted from higher to lower energies when the undulator gap is closed. The resultant measurement data is similar to the spectral profile as in Fig. 6(a). The result is then analyzed to determine the optimum gap that corresponds to some specific $K$ value. This optimization process is repeated for all the undulator segments and several different $K$ values to calibrate the relation between

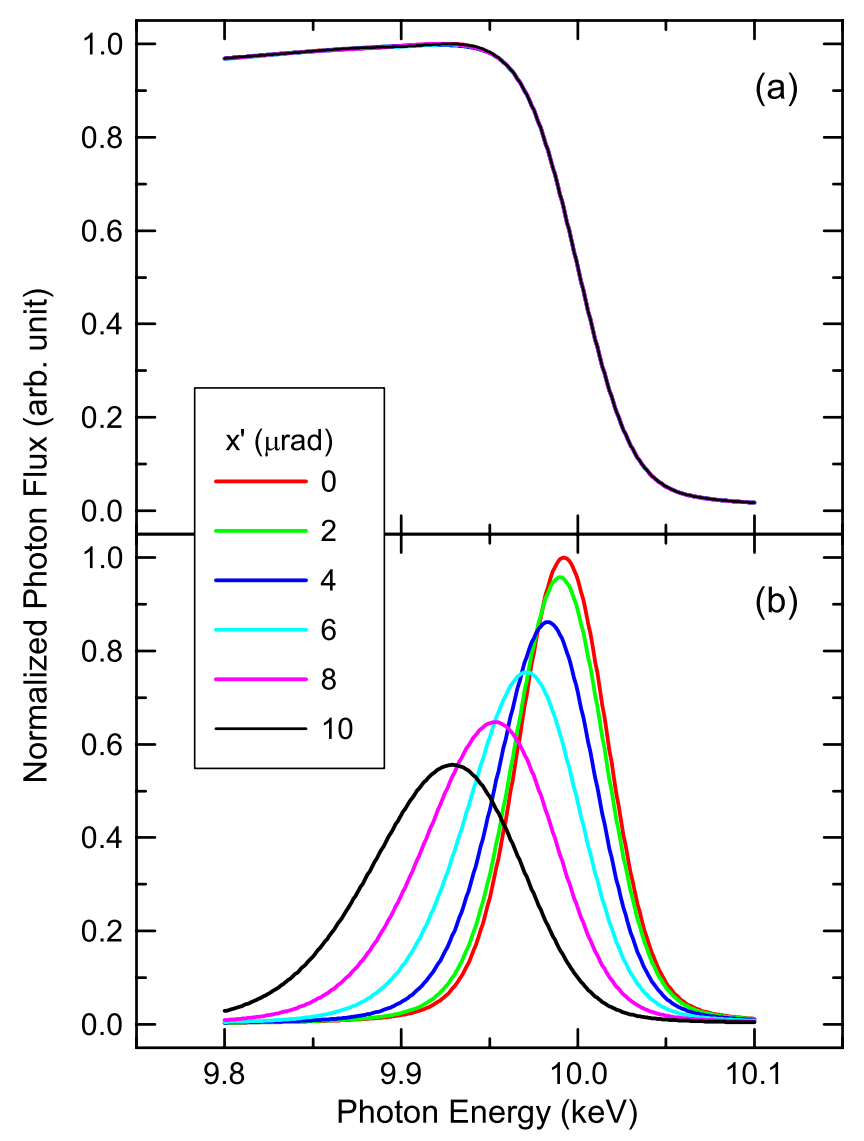

FIG. 6. Example of calculated spectra of spontaneous UR emitted from the 1st segment for two different slit aperture sizes: (a) $10 \mathrm{~mm}$ and (b) $0.5 \mathrm{~mm}$. 


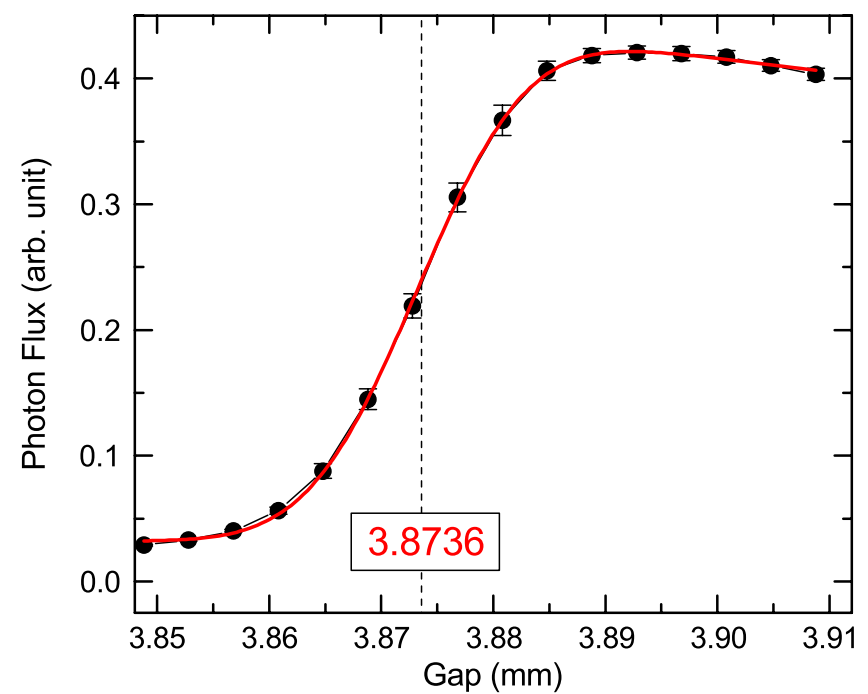

FIG. 7. Photon flux of spontaneous UR from the 1st segment measured at $10 \mathrm{keV}$ as a function of the gap (black circle). The fitting curve is also indicated (red line).

the gap and $K$ value. An example of the measurement result is shown in Fig. 7, in which the electron energy was fixed at $7.8 \mathrm{GeV}$ and the monochromator energy was fixed at $10 \mathrm{keV}$, and thus the $K$ value was nearly 2.1 .

The photon flux was found to drastically change around the gap between 3.88 and $3.87 \mathrm{~mm}$, roughly corresponding to the $K$ value of 2.1 . In order to exactly specify the optimum gap to give the $K$ value of 2.1, we introduced an empirical fitting function defined as

$$
f(g)=\left(a_{1}+a_{2} g\right) \operatorname{erf}\left(\frac{a_{3}-g}{a_{4}}\right)+a_{5},
$$

where erf is the Gauss error function and $a_{1} \sim a_{5}$ are the fitting parameters, among which $a_{3}$ gives the central position of the spectral edge and thus this can be regarded to be the optimum gap. The red line in Fig. 7 indicates the fitting function and the optimum gap in this example was found to be $3.8736 \mathrm{~mm}$. Repeating this process, all the undulator segments can be precisely aligned to have the identical $K$ value within some tolerance. Note that the absolute accuracy of the $K$ value determination with this method depends on the calibration accuracy of the monochromator and electron energy. Although the monochromator can be calibrated very accurately by means of, e.g., x-ray absorption experiments, the electron energy measurement with an accuracy of the order of $10^{-4}$ would be difficult and in fact not necessary. What should be done in the undulator commissioning is to reduce the relative difference in $K$ value between the undulator segments but not to know the exact number of the $K$ value.

\section{Height position adjustment}

In order to align the undulator height and to eliminate the vertical offset between the electron beam and magnetic

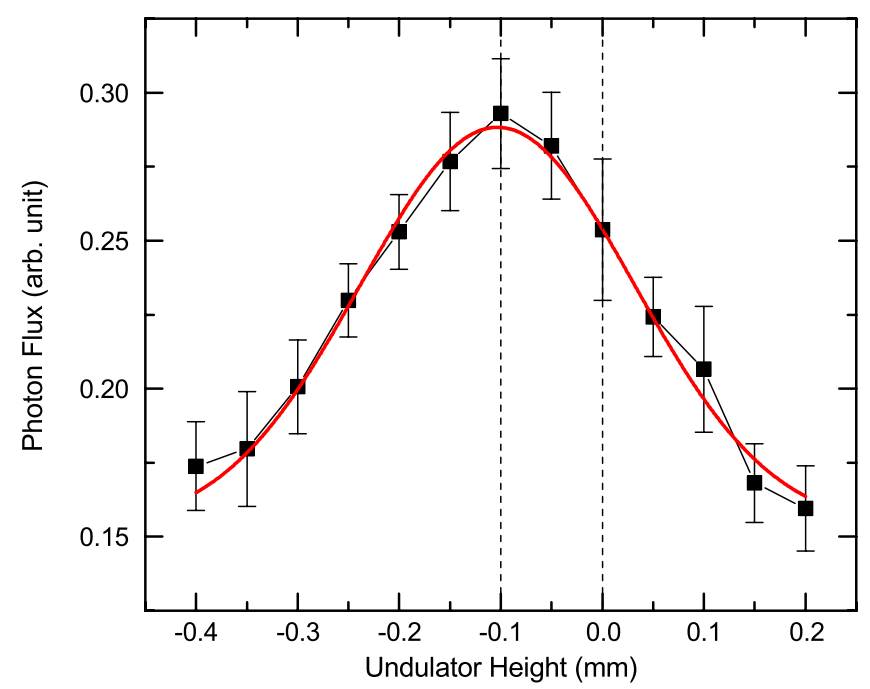

FIG. 8. Photon flux of spontaneous UR from the 1st segment measured at $10 \mathrm{keV}$ as a function of the undulator height (black square). The result of Gaussian fitting is also indicated (red line).

center of the undulator, the photon flux is measured as a function of the undulator height as in the gap distance adjustment. The $K$ value depends almost quadratically on the vertical offset under realistic conditions and thus the spectral edge is shifted from higher to lower energies when the offset increases. The photon flux is thus maximized when the offset vanishes if the gap and monochromator are set appropriately.

Figure 8 shows an example of the photon flux measured as a function of the undulator height. In this example, the undulator height was found to be misaligned by $0.1 \mathrm{~mm}$, which was corrected by a remotely controlled elevation system attached to the undulator.

\section{Phase matching}

In order to satisfy the phase matching condition, spectral characteristics specific to spontaneous UR emitted from two adjacent undulator segments have been utilized. Figure 9(a) shows the variation in spectra with the phase slippage between the 1 st and 2 nd segments, which was varied by changing the gap of the phase shifter installed in between. The undulator gap values of the two segments were set so that both the undulators had the identical $K$ value of 2.1. The spectral edge was found to become steeper at the phase shifter gap of $30.4 \mathrm{~mm}$ than at $32.4 \mathrm{~mm}$, meaning that this gap was closer to the optimum condition for the phase matching. In other words, the photon flux at $9.988 \mathrm{keV}$, as indicated by the dashed line in Fig. 9(a), was supposed to be maximum when the phase matching condition is satisfied. We have therefore measured the flux at that energy as a function of the phase shifter gap, the result of which is shown in Fig. 9(b). The phase slippage has been calculated from the magnetic measurement of the phase shifter and indicated in the 

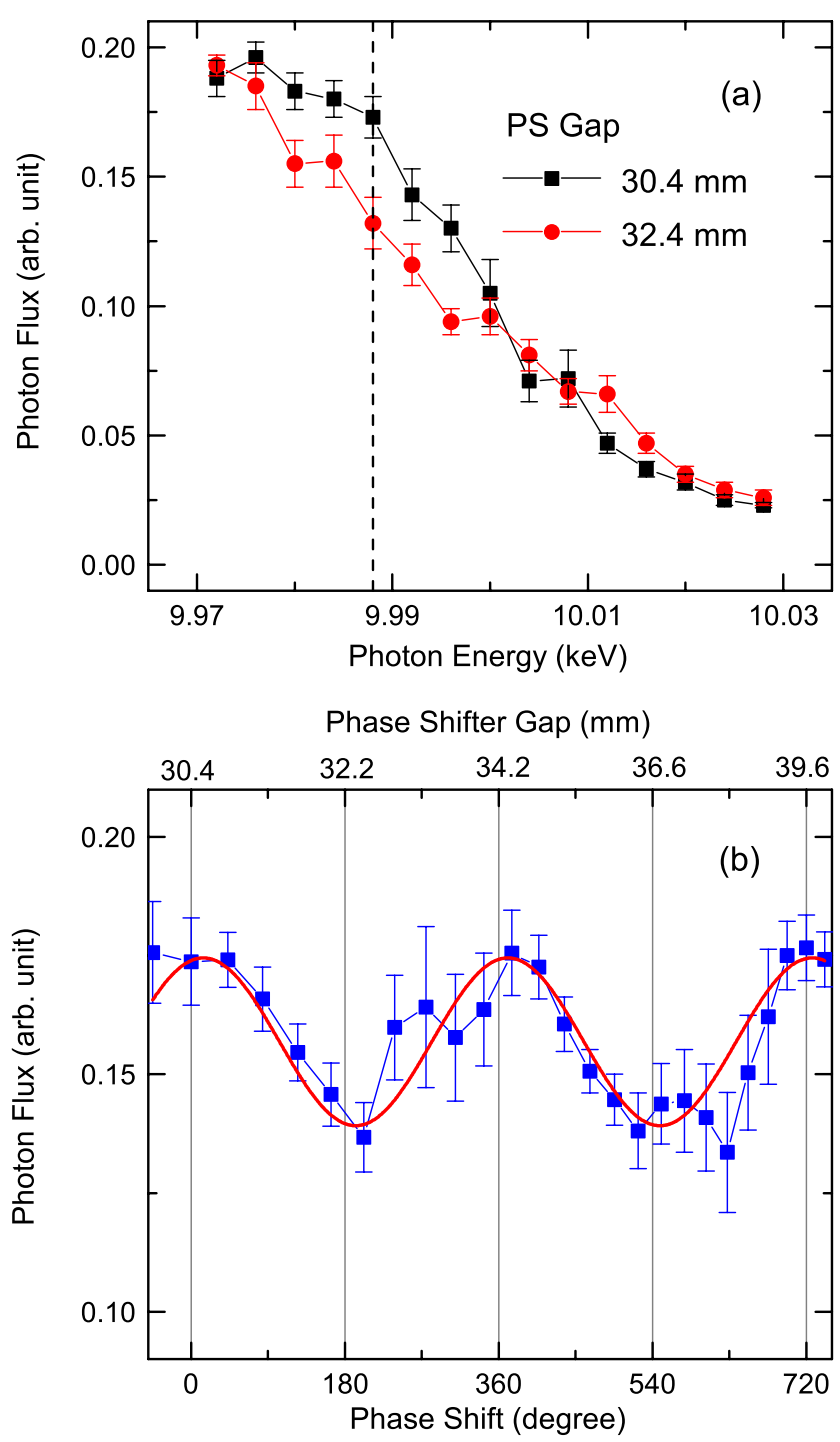

FIG. 9. Phase matching example. (a) Spectra of spontaneous UR emitted from the 1st and 2nd segments for two different gap values of the phase shifter installed in between. (b) Photon flux at $9.988 \mathrm{keV}$ measured as a function of the relative phase shift (blue square). The phase shifter gap corresponding to the phase shift of $n \pi$ is indicated in the top scale. The sinusoidal fitting of the measured result is also shown (red line).

relative value with respect to the phase slippage at the phase shifter gap of $30.4 \mathrm{~mm}$. The sinusoidal oscillation was found as expected, and the optimum phase shifter gap was found to be $30.4 \mathrm{~mm}$.

\section{E. Taper optimization}

The most straightforward way to compensate the wakefield effect is to optimize the undulator tapering so that the FEL intensity is maximized. In order to do so, it is necessary to get the FEL signal or at least the indication of FEL amplification, which is not very promising in the early stage of undulator commissioning. We therefore tried to measure the wakefield-induced energy loss by means of measuring the variation of the spontaneous UR spectrum for different wakefield conditions. For this purpose, the $K$ value and gap of the 17th undulator segment were fixed at 1.4 and $5.66 \mathrm{~mm}$, while those from the 1st to the 16th undulator segments were varied to change the wakefield condition. It is worth noting that such an operation to vary the wakefield condition is possible only for IVUs but not for the conventional out-vacuum devices.

The spectrum of spontaneous UR from the 17th undulator segment was measured to investigate the effects due to the wakefield. Note that the 18th segment was disabled by fully opening the gap because of the availability at the time of measurement. Also note that the electron beam was intensionally kicked by the steering magnet located just in
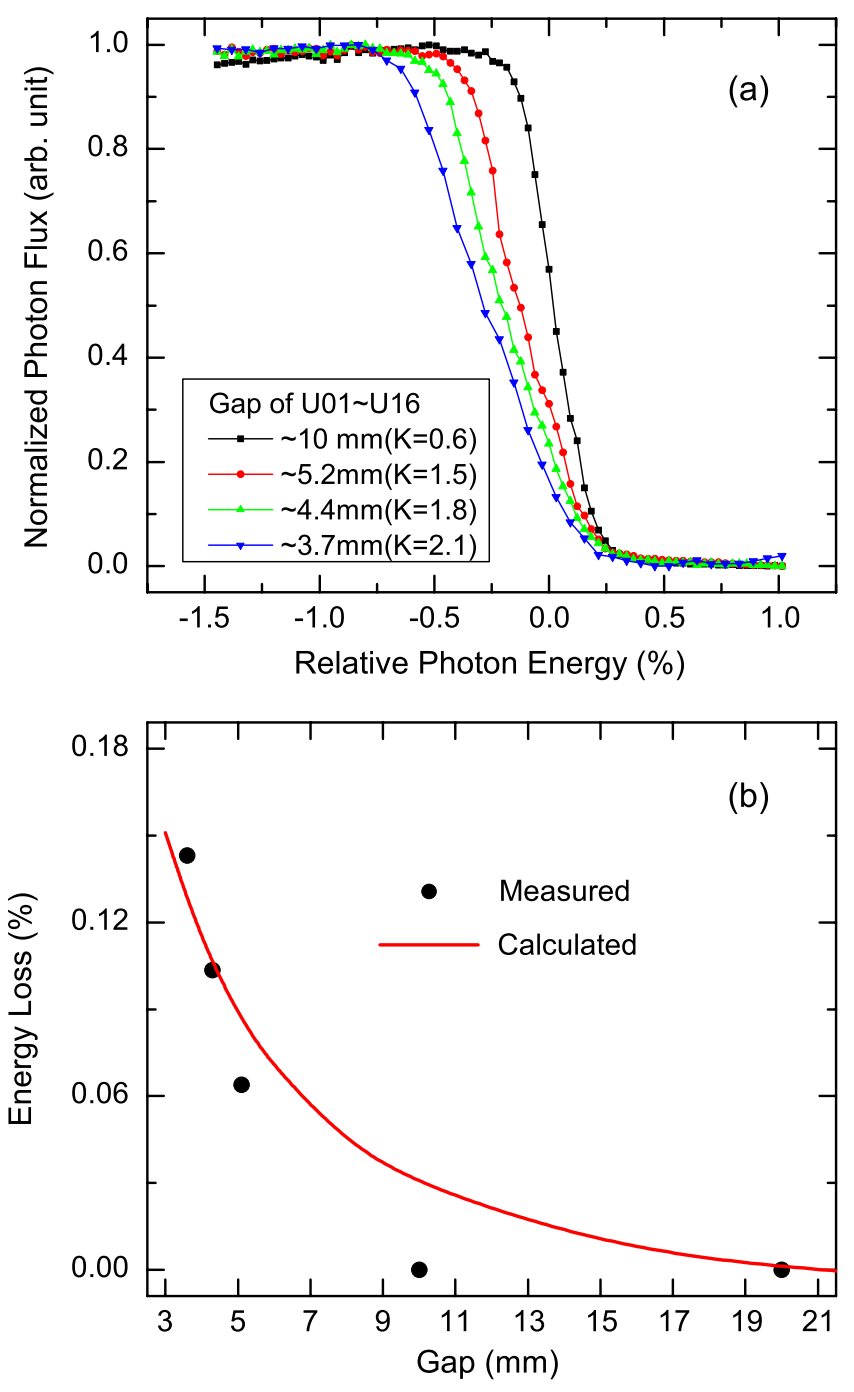

FIG. 10. Measurement results of the wakefield effect. (a) Spectral variation of spontaneous UR due to the wakefield condition. (b) Average energy loss by the wakefield as a function of the gap average over the upstream 16 segments, together with the estimation based on the measured temporal bunch profile and wakefield model. 
front of the 1st undulator segment to suppress the FEL process, in order to investigate the effects due to the wakefield alone. The measurement results are shown in Fig. 10(a).

The spectral edge was found to shift to lower energies and become more gradual when the wakefield was enhanced by closing the gap of the upstream 16 segments, meaning that the average electron energy decreased and energy spread increased. Figure 10(b) shows the variation of the average electron energy as a function of the gap values averaged over the upstream 16 segments. The calculated energy loss is also shown in Fig. 10(b), which was estimated from the temporal profile of the electron bunch measured by the rf deflector [12] and the mathematical form of the resistive wakefield in the parallel-plate configuration derived in [13]. It was found that the experimental and numerical results agreed well except for small discrepancies, which might be attributable to the fact that the temporal resolution of the bunch profile measurement was not enough to resolve the fine structure having impact on the wakefield characteristics. In any case, it is possible to roughly estimate the undulator taper to compensate the electron energy loss measured by the above procedure. For example, we need to apply an undulator taper of $-6 \times 10^{-5} / \mathrm{m}$ at the $K$ value of 2.1 , meaning that the undulator $K$ value should be decreased by $3 \times 10^{-4}$ from segment to segment. It is worth noting that this value is about $1 / 3$ of the optimum taper of $-10^{-3} /$ segment, which has been determined to maximize the FEL intensity as shown in Fig. 11. This discrepancy may be explained as follows.

It has been found that not the whole of the electron bunch contributes equally to lasing in SACLA [2]. To be more specific, there exists a region in the electron bunch, where the beam quality is much better than the projected

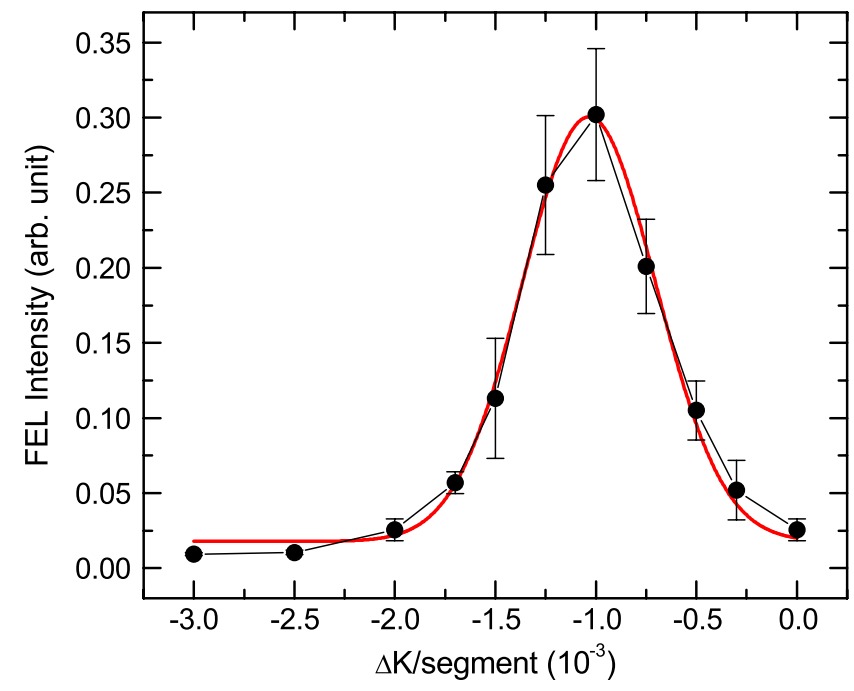

FIG. 11. Optimization of the undulator taper to maximize the FEL intensity. one. The undulator taper optimized to maximize the FEL intensity necessarily compensates the slice energy loss induced by the resistive wakefield at the bunch position where the good-quality region is located. On the other hand, the energy loss measured by the spontaneous UR spectrum is the projected one, which is not necessarily equal to the slice energy loss explained above. It is, however, still important to estimate the optimum taper by the spontaneous UR measurement to get the feeling of how much taper we need to apply, especially before getting an indication of lasing.

In SACLA, the photon energy can be tuned not only by changing the electron energy but also by changing the gap value. In the latter case, the wakefield condition is supposed to change as well, which is compensated by modifying the undulator taper to maximize the pulse energy of radiation.

\section{EVALUATION OF ALIGNMENT ACCURACY}

Now let us consider the achievable accuracy of alignment in the procedures described above. Needless to say, the accuracy can be in principle improved by increasing the number of shots for averaging at each data point. On the other hand, it is better to reduce the number of shots to save the time required for the alignment procedures and to avoid the possible ambiguity due to the long-term drift of accelerator operation. It is thus important to evaluate the expected alignment accuracy, when no averaging is made, i.e., only one shot is recorded at each data point. For the trajectory alignment, it is reasonable to define the alignment accuracy as the pointing stability of the photon beam given in Fig. 5. For the other alignment procedures, let us define the alignment accuracy as the deviation of the relevant fitting parameters in the functions to fit the measured data, as indicated by the red lines in Figs. 7, 8, 9(b), and 11.

As an example, the fluctuation of the fitting parameter $a_{3}$ in the fitting function given in Eq. (1), which determines the gap-alignment accuracy for the $K$-value tuning, was evaluated by simulating the shot-to-shot fluctuation of each data point based on the measured standard deviation indicated by the error bars in Fig. 7. Namely, a data set was generated according to

$$
f\left(g_{i}\right)=f_{0}\left(g_{i}\right)+\sigma_{f}\left(g_{i}\right) R_{i},
$$

where $g_{i}$ is the $i$ th gap for measurement, $f\left(g_{i}\right)$ is the expected value of photon flux measurement that will fluctuate from time to time, $f_{0}\left(g_{i}\right)$ and $\sigma_{f}\left(g_{i}\right)$ are the average and deviation of photon flux actually measured at the gap of $g_{i}$, and $R_{i}$ is again the Gaussian random number. By changing the seed for the random number generator, 100 data sets of the simulated flux measurements were generated. Then the optimum gap, namely the parameter $a_{3}$, was calculated by fitting each simulated data set. Figure 12 shows examples of the simulated flux measurements 


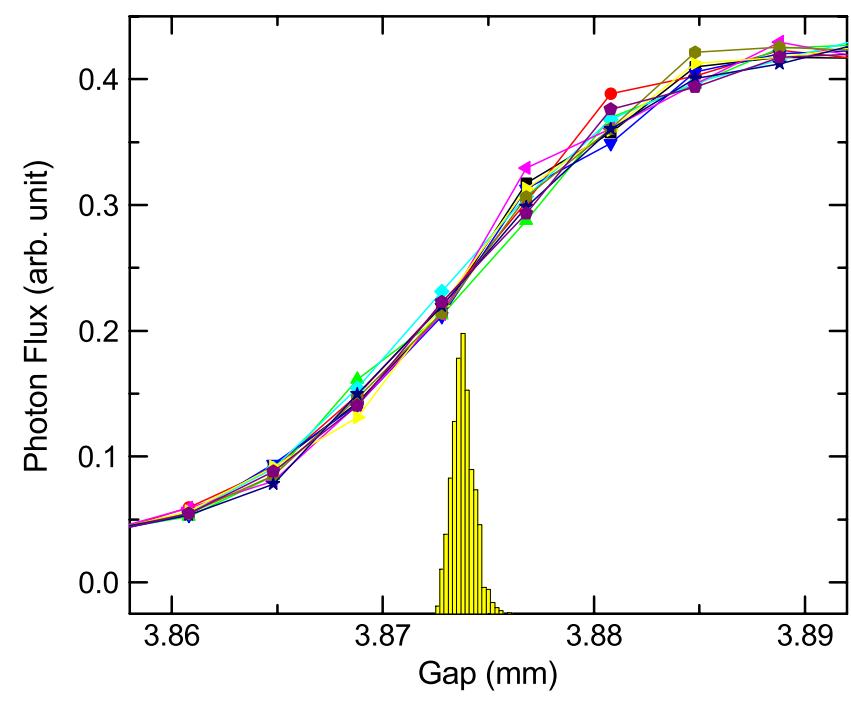

FIG. 12. Ten data sets of the simulated flux measurement and the histogram of the fitting parameter $a_{3}$, i.e., the optimum gap to give the $K$ value of 2.1 .

$f\left(g_{i}\right)$ (only 10 of 100 data sets are shown for clarity) and the histogram of the fitting parameter $a_{3}$, whose standard deviation was found to be $0.6 \mu \mathrm{m}$.

In the same manner, it is possible to calculate the standard deviations of the relevant fitting parameters (gap, height, phase, taper), which can be defined as the achieved accuracies of individual commissioning procedures. The results are summarized in Table II together with the pointing stability of the monochromatized spontaneous UR. In all the alignment items, the achieved accuracies are found to be better than the required values, showing the validity of the alignment schemes based on the characterization of $x$-ray radiation for the undulator commissioning in SACLA.

It should be noted that the fluctuation of the fitting parameter can be further reduced by increasing the number of shots for averaging. For example, the gap distance adjustment in SACLA is usually done by measuring the photon flux over 10 shots at each gap point to improve the alignment accuracy. The standard deviation of the optimum gap, which was obtained by repeating the gapalignment procedure by 10 times under the same condition, was found to be $0.36 \mu \mathrm{m}$, corresponding to the $K$-value deviation of as small as $8 \times 10^{-5}$.

The above discussion is validated by the fact that the pulse energy measured under the condition listed in Table I was nearly $0.19 \mathrm{~mJ}$, which is comparable to the ideal value calculated with the same beam parameters.

\section{SUMMARY}

The undulator commissioning procedures by means of characterization of radiation have been presented together with the actual results. The achieved alignment accuracies have been found to be within the tolerances. All of the alignment processes are now routinely in operation to offer the best performance to users in SACLA, whose frequencies depend on the target item. Among them, the trajectory alignment is being done most frequently, e.g., every two or three weeks, because the ground level of the SACLA undulator building, which is just 3 years old as of July 2012, is still moving slightly.

\section{ACKNOWLEDGMENTS}

The authors thank the SACLA operation team members for their continual support during the undulator commissioning. The authors also thank the SACLA beam line group members for development and maintenance of the photon diagnostics systems.

[1] P. Emma et al., Nat. Photonics 4, 641 (2010).

[2] T. Ishikawa et al., Nat. Photonics 6, 540 (2012).

[3] L.-H. Yu, S. Krinsky, R. L. Gluckstern, and J. B. J. van Zeijts, Phys. Rev. A 451163 (1992).

[4] T. Tanaka, H. Kitamura, and T. Shintake, Nucl. Instrum. Methods Phys. Res., Sect. A 528, 172 (2004).

[5] K. Halbach, J. Phys. C 1, 211 (1983).

[6] T. Tanaka, in Proceedings of the 26th FEL2004 Conference (Comitato Conferenze Elettra, Trieste, Italy, 2004), p. 2283, also refer to http://radiant.harima.riken .go.jp/simplex/.

[7] K. Tono, T. Kudo, M. Yabashi, T. Tachibana, Y. Feng, D. Fritz, J. Hastings, and T. Ishikawa, Rev. Sci. Instrum. 82, 023108 (2011).

[8] P. Emma, R. Carr, and H.D. Nuhn, Nucl. Instrum. Methods Phys. Res., Sect. A 429, 407 (1999).

[9] H.-D. Nuhn, in Proceedings of the 31st International Free Electron Laser Conference (FEL 09), Liverpool, UK (STFC Daresbury Laboratory, Warrington, 2009), p. 714.

[10] T. Kameshima et al. (unpublished).

[11] T. Tanaka and H. Kitamura, J. Synchrotron Radiat. 8, 1221 (2001), also refer to http://radiant.harima.riken.go.jp/ spectra/.

[12] H. Ego et al., in Proceedings of IPAC2011, San Sebastián, Spain (EPS-AG, Spain, 2011), p. 1221.

[13] K. L. F. Bane and G. Stupakov, Report No. SLAC-PUB10707, 2004. 